NISSUNA UMANA INVESTIGAZIONE SI PUO DIMANDARE VERA SCIENZIA S'ESSA NON PASSA PER LE MATEMATICHE DIMOSTRAZIONI LEONARDO DA VINCI

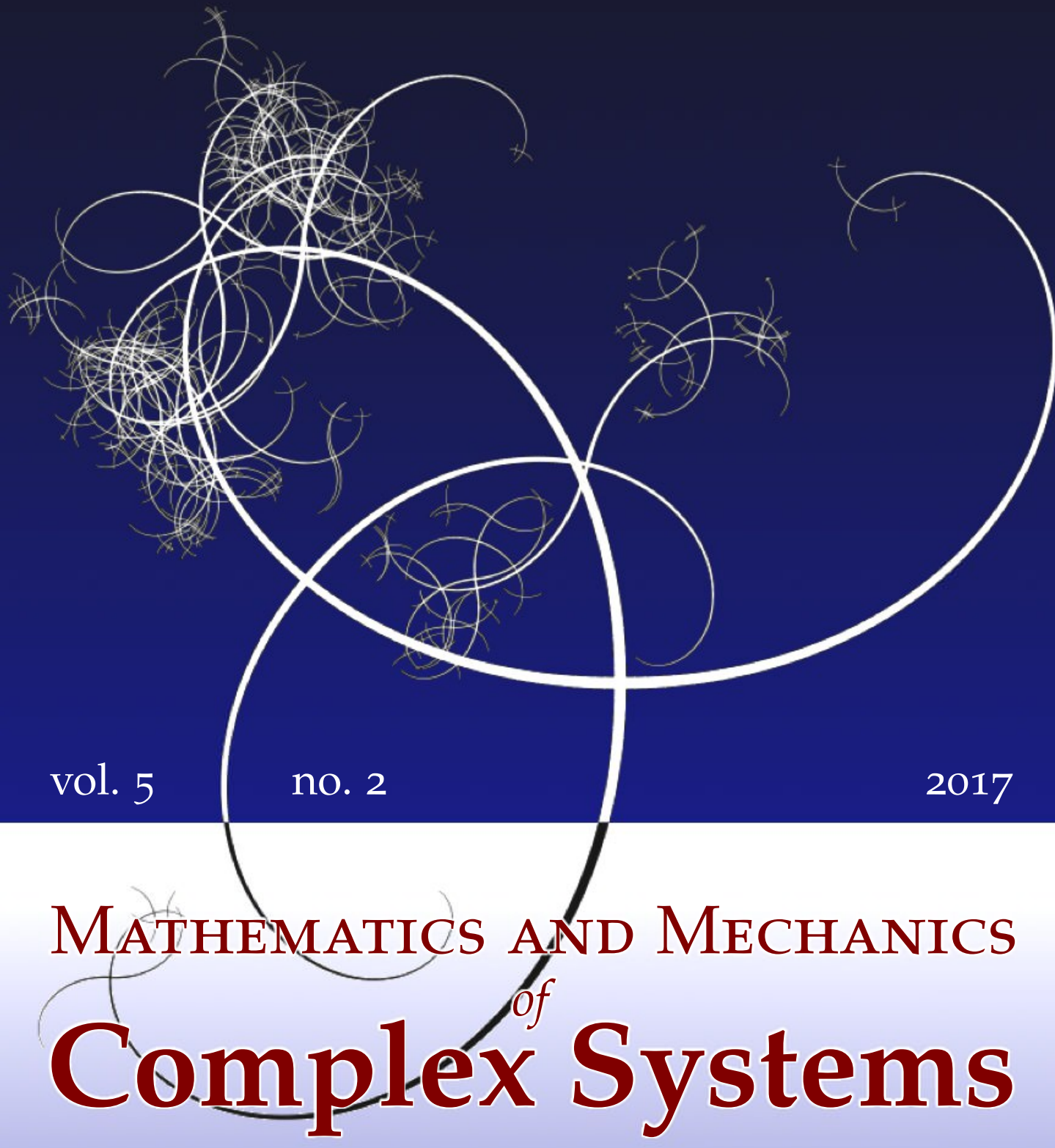

Ana Cristina Barroso, José Matias,

MARCO MORANDOTTI AND DAVID R. OWEN

EXPLICIT FORMULAS FOR RELAXED DISARRANGEMENT DENSITIES ARISING FROM STRUCTURED DEFORMATIONS 


\title{
EXPLICIT FORMULAS FOR RELAXED DISARRANGEMENT DENSITIES ARISING FROM STRUCTURED DEFORMATIONS
}

\author{
Ana Cristina Barroso, José Matias, \\ MARCO MORANDOTTI AND DAVID R. OWEN
}

In this paper we derive explicit formulas for disarrangement densities of submacroscopic separations, switches, and interpenetrations in the context of firstorder structured deformations. Our derivation employs relaxation within one mathematical setting for structured deformations of a specific, purely interfacial density, and the formula we obtain agrees with one obtained earlier in a different setting for structured deformations. Coincidentally, our derivation provides an alternative method for obtaining the earlier result, and we establish new explicit formulas for other measures of disarrangements that are significant in applications.

1. Introduction

163

2. Proof of the upper-bound inequality 177

3. Aside on isotropic vectors 180

4. Completion of the proof of the upper-bound inequality 182

5. Additional explicit formulas for disarrangement densities 184

$\begin{array}{ll}\text { Acknowledgments } & 187\end{array}$

$\begin{array}{ll}\text { References } & 188\end{array}$

\section{Introduction}

Structured deformations provide a multiscale geometry that captures the contributions at the macrolevel of both smooth geometrical changes and nonsmooth geometrical changes (disarrangements) at submacroscopic levels. For each (first-order) structured deformation $(g, G)$ of a continuous body, the tensor field $G$ is known to be a measure of deformations without disarrangements, and $M:=\nabla g-G$ is known to be a measure of deformations due to disarrangements. The tensor fields $G$ and $M$ together deliver not only standard notions of plastic deformation, but $M$ and its curl deliver the Burgers vector field associated with closed curves in the body

\section{Communicated by Miroslav Šilhavý.}

MSC2010: primary 49J45; secondary 74A60, 74G65, 15A99.

Keywords: structured deformations, relaxation, disarrangements, interfacial density, bulk density, isotropic vectors. 
and the dislocation density field used in describing geometrical changes in bodies with defects. Recently, Owen and Paroni [2015] explicitly evaluated some relaxed energy densities arising in Choksi and Fonseca's [1997] energetics of structured deformations and thereby showed (1) $(\operatorname{tr} M)^{+}$, the positive part of $\operatorname{tr} M$, is a volume density of disarrangements due to submacroscopic separations, (2) $(\operatorname{tr} M)^{-}$, the negative part of $\operatorname{tr} M$, is a volume density of disarrangements due to submacroscopic switches and interpenetrations, and (3) $|\operatorname{tr} M|$, the absolute value of $\operatorname{tr} M$, is a volume density of all three of these nontangential disarrangements: separations, switches, and interpenetrations. The main contribution of the present research is to show that a different approach to the energetics of structured deformations, that due to Baía, Matias, and Santos [Baía et al. 2012], confirms the roles of $(\operatorname{tr} M)^{+}$, $(\operatorname{tr} M)^{-}$, and $|\operatorname{tr} M|$ established by Owen and Paroni. In doing so, we give an alternative, shorter proof of Owen and Paroni's results, and we establish additional explicit formulas for other measures of disarrangements.

To motivate our study and to provide necessary background, we briefly discuss in the following subsections of this introduction some concepts and results from the multiscale geometry of structured deformations. (Readers familiar with this material may wish to skip to Section 1E, where our main results are summarized.)

\section{A. Structured deformations and disarrangement densities in the setting of Del}

Piero and Owen. The need in continuum mechanics to include the effects of multiscale geometrical changes led Del Piero and Owen [1993] to a notion of structured deformations as triples $(\kappa, g, G)$, where

- the injective, piecewise continuously differentiable field $g$ maps the points of a continuous body into physical space and describes macroscopic changes in the geometry of the body,

- the piecewise continuous tensor field $G$ maps the body into the space of linear mappings on the translation space of physical space and satisfies the "accommodation inequality"

$$
0<C<\operatorname{det} G(x) \leq \operatorname{det} \nabla g(x) \quad \text { at each point } x,
$$

where $\nabla$ denotes the classical gradient operator, and

- $\kappa$ is a surface-like subset of the body that describes preexisting, unopened macroscopic cracks.

A geometrical interpretation of the field $G$ is provided by the approximation theorem [Del Piero and Owen 1993]: for each structured deformation $(\kappa, g, G)$, there exists a sequence of injective, piecewise smooth deformations $f_{n}$ and a sequence 
of surface-like subsets $\kappa_{n}$ of the body such that

$$
\begin{aligned}
g & =\lim _{n \rightarrow \infty} f_{n}, \\
G & =\lim _{n \rightarrow \infty} \nabla f_{n},
\end{aligned}
$$

and

$$
\kappa=\bigcup_{n=1}^{\infty} \bigcap_{p=n}^{\infty} \kappa_{p}
$$

The limits in (1-2) and (1-3) are taken in the sense of $L^{\infty}$ convergence. A sequence $n \mapsto f_{n}$ of piecewise smooth, injective functions satisfying (1-2) and (1-3) is called a determining sequence for the pair $(g, G)$, and each term $f_{n}$ is interpreted as describing the body divided into tiny pieces that may individually undergo smooth geometrical changes and that also may undergo disarrangements, i.e., may separate or slide relative to each other. In this context, we write $f_{n} \rightsquigarrow(g, G)$. From (1-3) we see that $G$ captures the effects at the macrolevel of smooth geometrical changes at submacroscopic levels, and we call $G$ the deformation without disarrangements.

Del Piero and Owen [1995] proved that for every structured deformation $(\kappa, g, G)$, for every determining sequence $n \mapsto f_{n}$ for $(g, G)$, and for every point $x$ where $g$ is differentiable and where $G$ is continuous there holds

$$
\lim _{r \rightarrow 0} \lim _{n \rightarrow \infty} \frac{\int_{J\left(f_{n}\right) \cap B_{r}(x)}\left[f_{n}\right](y) \otimes v(y) d \mathscr{H}^{N-1}(y)}{\left|B_{r}(x)\right|}=\nabla g(x)-G(x) .
$$

Here, $\mathscr{H}^{N-1}$ denotes the $(N-1)$-dimensional Hausdorff measure on $\mathbb{R}^{N}, B_{r}(x)$ denotes the open ball centered at $x$ of radius $r,\left|B_{r}(x)\right|$ denotes its volume (i.e., its $N$-dimensional Lebesgue measure), $J\left(f_{n}\right)$ denotes the jump set of $f_{n}$ (i.e., points where $f_{n}$ can suffer jump discontinuities), and $\left[f_{n}\right](y) \otimes v(y)$ is the tensor product of the jump [ $f_{n}$ ] of $f_{n}$ with the normal $v$ to the jump set. This result permits us to call the tensor

$$
M(x):=\nabla g(x)-G(x)
$$

the deformation due to disarrangements because it captures, in the limit as $n$ tends to infinity, the volume density of separations and slips between pieces of the body as described by the approximating deformations $f_{n}$. We may then regard the tensor field $M$ as a tensorial disarrangement density that, for every determining sequence $n \mapsto f_{n}$ for $(g, G)$, reflects the limits of interfacial discontinuities of the approximating deformations $f_{n}$. Moreover, (1-2) and (1-3) along with the definition of $M(1-5)$ yield the alternative formula for the disarrangement density:

$$
M=\nabla\left(\lim _{n \rightarrow \infty} f_{n}\right)-\lim _{n \rightarrow \infty} \nabla f_{n} .
$$


Consequently, $M$ quantitatively measures the lack of commutativity of the classical gradient $\nabla$ and the limit operator $\lim _{n \rightarrow \infty}$ for $L^{\infty}$ convergence.

The trivial algebraic relation

$$
\nabla g=G+M
$$

together with the identification relations (1-3) and (1-4) shows that the macroscopic deformation gradient $\nabla g$ has an additive decomposition into its part $G$ without disarrangements and its part $M$ due to disarrangements. Because $G$ has invertible values, (1-6) leads immediately to two multiplicative decompositions for $\nabla g$ :

$$
\nabla g=G\left(I+G^{-1} M\right)=\left(I+M G^{-1}\right) G .
$$

The disarrangement density $M$ and the deformation without disarrangements $G$ have an additional property significant in the description of defects and dislocations in a continuous body in three dimensions. We consider a smooth surface $\mathscr{S}$ with smooth bounding closed curve $\gamma$, both contained in a region in the body where $g$ and $G$ are smooth. The relation (1-6) and the smoothness of $g$ imply

$$
0=\oint_{\gamma} \nabla g(x) d x=\oint_{\gamma} G(x) d x+\oint_{\gamma} M(x) d x .
$$

The vector $\oint_{\gamma} M(x) d x$ measures the displacement due to disarrangements along $\gamma$ and may be called the Burgers vector [Del Piero and Owen 1993] for $\gamma$ arising from the given structured deformation. Application of Stokes' theorem to $\oint_{\gamma} G(x) d x$ and $\oint_{\gamma} M(x) d x$ and use of the previous relation yield the formulas for the Burgers vector:

$$
\oint_{\gamma} M(x) d x=\int_{\mathscr{S}} \operatorname{curl} M(x) v(x) d A_{x}=-\int_{\mathscr{S}} \operatorname{curl} G(x) v(x) d A_{x} .
$$

The second-order tensor field curl $M=-\operatorname{curl} G$ thus determines the Burgers vector associated with $\gamma$ for every closed curve and corresponds to familiar measures of dislocation density [Kröner 1958; Nye 1953]. In this manner, the disarrangement density tensor $M$ determines both the Burgers vector and the dislocation density tensor, both basic tools in modeling the effects of submacroscopic defects on the response of solids.

The tensorial relations (1-5) and (1-4) yield upon application of the trace operator the scalar relation

$$
\lim _{r \rightarrow 0} \lim _{n \rightarrow \infty} \frac{\int_{J\left(f_{n}\right) \cap B_{r}(x)}\left[f_{n}\right](y) \cdot v(y) d \mathscr{H}^{N-1}(y)}{\left|B_{r}(x)\right|}=\operatorname{tr} M(x)
$$

in which $\left[f_{n}\right](y) \cdot v(y)$ is the scalar product of the jump and of the normal at $y$. The formula (1-7) tells us that $\operatorname{tr} M$ is a scalar (bulk) disarrangement density that captures the components of the jumps of $f_{n}$ that are normal to the jump set. Moreover, 
this scalar disarrangement density at $x, \operatorname{tr} M(x)$, allows for cancellation of positive and negative contributions of $\left[f_{n}\right](y) \cdot v(y)$ at points $y$ near $x$ to the integral on the left-hand side of (1-7). Thus, $\operatorname{tr} M(x)$ does not distinguish between jumps with $\left[f_{n}\right](y) \cdot v(y)>0$ that pull apart small pieces of the body near $x$ and jumps with $\left[f_{n}\right](y) \cdot v(y)<0$ that cause small pieces near $x$ to switch places. Because the approximating $f_{n}$ are injective, the possibility for the case $\left[f_{n}\right](y) \cdot v(y)<0$ that $f_{n}$ can cause adjacent small pieces of the body to interpenetrate is ruled out.

Owen and Paroni [2015] refined the scalar disarrangement density $\operatorname{tr} M$ by replacing $\left[f_{n}\right](y) \cdot v(y)$ by its positive part throughout the jump set of $f_{n}$ or by its negative part throughout the jump set:

$$
\begin{aligned}
& \left(\left[f_{n}\right](y) \cdot v(y)\right)^{+}=\frac{1}{2}\left(\left|\left[f_{n}\right](y) \cdot v(y)\right|+\left[f_{n}\right](y) \cdot v(y)\right), \\
& \left(\left[f_{n}\right](y) \cdot v(y)\right)^{-}=\frac{1}{2}\left(\left|\left[f_{n}\right](y) \cdot v(y)\right|-\left[f_{n}\right](y) \cdot v(y)\right) .
\end{aligned}
$$

The field $\left(\left[f_{n}\right] \cdot v\right)^{+}$on the jump set is a scalar (interfacial) disarrangement density that measures separations of pieces of the body caused by $f_{n}$ while the field $\left(\left[f_{n}\right] \cdot v\right)^{-}$is a scalar (interfacial) disarrangement density that measures the switching of pieces of the body caused by $f_{n}$. Since

$$
\left|\left[f_{n}\right](y) \cdot v(y)\right|=\left(\left[f_{n}\right](y) \cdot v(y)\right)^{+}+\left(\left[f_{n}\right](y) \cdot v(y)\right)^{-},
$$

the field $\left|\left[f_{n}\right](y) \cdot v(y)\right|$ is a scalar disarrangement density that measures both separations and switches. We fix a part $\mathscr{P}$ of the body, integrate (1-8) or (1-9) over $J\left(f_{n}\right) \cap \mathscr{P}$, and use the formula (1-7) to obtain the relations

$$
\begin{aligned}
& \liminf _{n \rightarrow \infty} \int_{J\left(f_{n}\right) \cap \mathscr{P}}\left(\left[f_{n}\right](y) \cdot v(y)\right)^{ \pm} d \mathscr{H}^{N-1}(y) \\
& =\frac{1}{2} \liminf _{n \rightarrow \infty} \int_{J\left(f_{n}\right) \cap \mathscr{P}}\left|\left[f_{n}\right](y) \cdot v(y)\right| d \mathscr{H}^{N-1}(y) \\
& \quad \pm \frac{1}{2} \liminf _{n \rightarrow \infty} \int_{J\left(f_{n}\right) \cap \mathscr{P}}\left[f_{n}\right](y) \cdot v(y) d \mathscr{H}^{N-1}(y) \\
& =\frac{1}{2} \liminf _{n \rightarrow \infty} \int_{J\left(f_{n}\right) \cap \mathscr{P}}\left|\left[f_{n}\right](y) \cdot v(y)\right| d \mathscr{H}^{N-1}(y) \pm \frac{1}{2} \int_{\mathscr{P}} \operatorname{tr} M(x) d \mathscr{L}^{N}(x) .
\end{aligned}
$$

Consequently, the limiting behavior of the integral of $\left(\left[f_{n}\right](y) \cdot v(y)\right)^{ \pm}$in (1-10) as $n$ tends to $\infty$ is determined by the behavior of the integral of $\left|\left[f_{n}\right](y) \cdot v(y)\right|$, and we restrict our attention to the latter. We expect that

$$
\liminf _{n \rightarrow \infty} \int_{J\left(f_{n}\right) \cap \mathscr{P}}\left|\left[f_{n}\right](y) \cdot v(y)\right| d \mathscr{H}^{N-1}(y),
$$

unlike

$$
\liminf _{n \rightarrow \infty} \int_{J\left(f_{n}\right) \cap \Im}\left[f_{n}\right](y) \cdot v(y) d \mathscr{H}^{N-1}(y)
$$


will depend upon the choice of determining sequence for $(g, G)$. Therefore, we are led to consider the most economical manner in which separations and switches can arise among the determining sequences for $(g, G)$ :

$$
\begin{aligned}
\mathscr{V}^{|\cdot|}(g, G & ; \mathscr{P}) \\
& :=\inf \left\{\liminf _{n \rightarrow \infty} \int_{J\left(f_{n}\right) \cap \mathscr{P}}\left|\left[f_{n}\right](y) \cdot v(y)\right| d \mathscr{H}^{N-1}(y): f_{n} \rightsquigarrow(g, G)\right\} .
\end{aligned}
$$

The number $\mathscr{V}^{|\cdot|}(g, G ; \mathscr{P})$ so defined has the dimension of volume, and we call $\mathscr{V}^{l \cdot \mid}(g, G ; \mathscr{P})$ the (minimal) volume swept out by disarrangements in $\mathscr{P}$ for $(g, G)$. If we replace $|\cdot|$ everywhere in (1-11) by "+" or everywhere by "-", then we call the number $\mathscr{V}^{+}(g, G ; \mathscr{P})$ the (minimal) volume swept out by separations in $\mathscr{P}$ for $(g, G)$ and the number $\mathscr{V}^{-}(g, G ; \mathscr{P})$ the (minimal) volume swept out by switches in $\mathscr{P}$ for $(g, G)$. The formulas (1-10) imply the simple formulas

$$
\mathscr{V}^{ \pm}(g, G ; \mathscr{P})=\frac{1}{2} \mathscr{V}^{|\cdot|}(g, G ; \mathscr{P}) \pm \frac{1}{2} \int_{\mathscr{P}} \operatorname{tr} M(x) d \mathscr{L}^{N}(x)
$$

and, in view of the form of the second term on the right-hand side, raise the following basic question: does the volume swept out by disarrangements $\mathscr{V}^{|\cdot|}(g, G ; \mathscr{P})$ as defined in (1-11) have an associated disarrangement density which, when integrated over $\mathscr{P}$, recovers $\mathscr{V}^{\mid} \cdot \mid(g, G ; \mathscr{P})$ ? If so, what specific information can be obtained about the dependence of the integrand upon the structured deformation $(g, G)$ ?

While the setting for structured deformations described in this subsection is quite suitable for formulating refined field equations in continuum mechanics [Deseri and Owen 2003] that reflect the influence of submacroscopic geometrical changes in a body, this setting has not provided answers to questions such as the ones just raised. Part of the difficulty with the setting provided in [Del Piero and Owen 1993] lies in the choice of smoothness placed on $g$ and its approximates $f_{n}$ while another part lies in the requirement that $g$ and $f_{n}$ be injective. An alternative setting provided by Choksi and Fonseca [1997] was proposed for dealing with such questions and is described briefly in the next subsection.

\section{B. Structured deformations and disarrangement densities in Choksi and Fon-} seca's setting. We describe here a few essential elements of the treatment of structured deformations by Choksi and Fonseca [1997]. The articles [Choksi et al. 1999; Baía et al. 2012; 2011; Šilhavý 2015] also provide summaries of that treatment, and [Baía et al. 2012; 2011; Šilhavý 2015] provide alternative settings for structured deformations. The summary in [Choksi et al. 1999] is intended for those interested in immediate applications in continuum mechanics while [Baía et al. 2012] sets the stage for applications of structured deformations to thin bodies [Matias and Santos 2014]. The article [Šilhavý 2015] reexamines the results of [Choksi and 
Fonseca 1997] in a broader setting while providing refinements of counterparts of the approximation theorem and the identification relation (1-4).

According to Choksi and Fonseca, a structured deformation is a pair $(g, G)$ in which $g: \Omega \rightarrow \mathbb{R}^{N}$, with $\Omega$ an open subset of the space $\mathbb{R}^{N}$ of $N$-tuples of real numbers, and $G: \Omega \rightarrow \mathbb{R}^{N \times N}$, with $\mathbb{R}^{N \times N}$ the space of $N \times N$ matrices with real entries. The mapping $G$ is assumed to be integrable on $\Omega, G \in L^{1}\left(\Omega ; \mathbb{R}^{N \times N}\right)$, and $g$ is assumed to be in the space $S B V\left(\Omega ; \mathbb{R}^{N}\right)$, i.e., is a function of bounded variation with the additional property that its distributional derivative $D g$, as a bounded measure, has zero Cantor part:

$$
D g=\nabla g \mathscr{L}^{N}+[g] \otimes \nu \mathscr{H}^{N-1} .
$$

Here the integrable mapping $\nabla g$ is the density of the absolutely continuous part $\nabla g \mathscr{L}^{N}$ of $D g$ with respect to $N$-dimensional Lebesgue measure $\mathscr{L}^{N}$, and $[g] \otimes v$ is the density of the singular part $[g] \otimes \nu \mathcal{H}^{N-1}$ of $D g$ with respect to $(N-1)$ dimensional Hausdorff measure $\mathscr{H}^{N-1}$. The singular part is concentrated on $J(g)$, the jump set of $g$, and as usual, $[g]$ denotes the jump in $g$ and $v$ denotes the normal to the jump set $J(g)$. It is important to note that $\nabla g$ in the present setting is no longer the classical gradient of a smooth field and, consequently, need not be curlfree. Nevertheless, $\nabla g$ satisfies an integral version of the property of approximation by linear mappings that defines the classical gradient of smooth fields.

Choksi and Fonseca [1997] prove a version of the approximation theorem with approximating deformations $f_{n}$ also in $\operatorname{SBV}\left(\Omega ; \mathbb{R}^{N}\right)$ and with (1-2) and (1-3) replaced respectively by

$$
f_{n} \rightarrow g \quad \text { in } L^{1}\left(\Omega ; \mathbb{R}^{N}\right)
$$

and

$$
\nabla f_{n} \rightarrow G \quad \text { weakly in the sense of measures. }
$$

We note that no restriction in the form of the accommodation inequality (1-1) or in the form of a requirement of injectivity of $g$ or $f_{n}$ is imposed in the present context. We again use the term determining sequence to describe a sequence $n \mapsto f_{n}$ satisfying (1-13) and (1-14) for a given structured deformation $(g, G)$, and we again write $f_{n} \rightsquigarrow(g, G)$ when (1-13) and (1-14) both hold. The properties of distributional derivatives along with relations (1-12), (1-13), and (1-14) justify the calculation

$$
\begin{aligned}
\nabla g \mathscr{L}^{N}+[g] \otimes \nu \mathscr{H}^{N-1} & =D \lim _{n \rightarrow \infty} f_{n}=\lim _{n \rightarrow \infty} D f_{n} \\
& =\lim _{n \rightarrow \infty}\left(\nabla f_{n} \mathscr{L}^{N}+\left[f_{n}\right] \otimes \nu \mathcal{H}^{N-1}\right) \\
& =G \mathscr{L}^{N}+\lim _{n \rightarrow \infty}\left(\left[f_{n}\right] \otimes \nu \mathscr{H}^{N-1}\right),
\end{aligned}
$$

where the convergence indicated in the last three lines is weak convergence in the sense of measures. We conclude that the singular parts $\left[f_{n}\right] \otimes v \mathscr{H}^{N-1}$ of the 
approximating deformations $f_{n}$ converge in the same sense and that their limit satisfies

$$
\lim _{n \rightarrow \infty}\left(\left[f_{n}\right] \otimes v \mathscr{H}^{N-1}\right)=(\nabla g-G) \mathscr{L}^{N}+[g] \otimes \nu \mathscr{H}^{N-1} .
$$

In particular, the restriction of the limiting measure $\lim _{n \rightarrow \infty}\left(\left[f_{n}\right] \otimes v \mathscr{H}^{N-1}\right)$ to the complement of the jump set $J(g)$ agrees with the corresponding restriction of $(\nabla g-G) \mathscr{L}^{N}=M \mathscr{L}^{N}$. Consequently, the tensor field $M=\nabla g-G$ retains in this broader setting for structured deformations its identity as a tensor density of disarrangements for $(g, G)$. The formula (1-15) shows that when $M=\nabla g-G \neq 0$, while all of the measures $\left[f_{n}\right] \otimes v \mathscr{H}^{N-1}$ are supported on sets $J\left(f_{n}\right)$ of $\mathscr{L}^{N}$-measure zero and so have $\mathscr{L}^{N}$-parts zero, the limit measure $\lim _{n \rightarrow \infty}\left(\left[f_{n}\right] \otimes v \mathscr{H}^{N-1}\right)$ has $\mathscr{L}^{N}$-part $M \mathscr{L}^{N}$ nonzero. This observation points to the fact that the jump sets $J\left(f_{n}\right)$ can diffuse in the limit throughout the domain $\Omega$ so that the limiting measure $\lim _{n \rightarrow \infty}\left(\left[f_{n}\right] \otimes v \mathcal{H}^{N-1}\right)$ is supported in part on sets of positive $\mathscr{L}^{N}$-measure. This provides a counterpart in the $S B V$-setting to the relation (1-4) in which the limit of jumps on the left-hand side delivers the $\mathscr{L}^{N}$-density $M$. (See [Šilhavý 2015] for a detailed derivation of a counterpart of (1-4) in a somewhat broader setting for structured deformations than $S B V$.)

We briefly note that the scalar density of disarrangements $\operatorname{tr} M=\operatorname{tr}(\nabla g-G)$ that counts only normal components of jumps and that emerged in the previous setting also appears in the present setting when one takes the trace of every member of (1-15): if $f_{n} \rightsquigarrow(g, G)$, then

$$
\lim _{n \rightarrow \infty}\left(\left[f_{n}\right] \cdot v \mathscr{H}^{N-1}\right)=\operatorname{tr}(\nabla g-G) \mathscr{L}^{N}+[g] \cdot v \mathscr{H}^{N-1} .
$$

However, as was the case in the setting of Del Piero and Owen, replacement of $\left[f_{n}\right] \cdot v$ by $\left(\left[f_{n}\right] \cdot v\right)^{ \pm}$or by $\left|\left[f_{n}\right] \cdot v\right|$ need not yield a limit of the corresponding measures and, if a limit exists, the limit may depend upon the choice of determining sequence $n \mapsto f_{n}$. The setting of Choksi and Fonseca was formulated as a means of resolving these difficulties, and we summarize some aspects of that resolution in the next subsection.

1C. Relaxation of energies for structured deformations. In Section 1A we introduced the optimal function

$$
\mathscr{V}^{|\cdot|}(g, G ; \mathscr{P})=\inf \left\{\liminf _{n \rightarrow \infty} \int_{J\left(f_{n}\right) \cap \mathscr{P}}\left|\left[f_{n}\right](y) \cdot v(y)\right| d \mathscr{H}^{N-1}(y): f_{n} \rightsquigarrow(g, G)\right\} .
$$

Optimal functions arising from structured deformations can be analyzed using the results of Choksi and Fonseca [1997] on "relaxation of energies" for structured deformations. In that approach, the integral $\int_{J\left(f_{n}\right) \cap \mathscr{P}}\left|\left[f_{n}\right](y) \cdot v(y)\right| d \mathscr{H}^{N-1}(y)$ is 
replaced by an initial energy functional

$$
E\left(f_{n}\right)=\int_{\Omega} W\left(\nabla f_{n}(y)\right) d \mathscr{L}^{N}(y)+\int_{J\left(f_{n}\right) \cap \Omega} \psi\left(\left[f_{n}\right](y), v(y)\right) d \mathscr{H}^{N-1}(y)
$$

defined for $f_{n} \in S B V\left(\Omega ; \mathbb{R}^{N}\right)$. By imposing conditions on the initial bulk energy density $W$ and on the initial interfacial energy density $\psi$, the goal is to obtain for the relaxed energy $I(g, G)$ defined by

$$
\begin{aligned}
I(g, G):=\inf \left\{\liminf _{n \rightarrow \infty}(\right. & \int_{\Omega} W\left(\nabla f_{n}(y)\right) d \mathscr{L}^{N}(y) \\
& \left.\left.+\int_{J\left(f_{n}\right) \cap \Omega} \psi\left(\left[f_{n}\right](y), v(y)\right) d \mathscr{H}^{N-1}(y)\right): f_{n} \rightsquigarrow(g, G)\right\}
\end{aligned}
$$

a representation of the form

$$
I(g, G)=\int_{\Omega} H(\nabla g(y), G(y)) d \mathscr{L}^{N}(y)+\int_{J(g) \cap \Omega} h([g](y), v(y)) d \mathscr{H}^{N-1}(y)
$$

and to deduce properties of the relaxed bulk energy density $H$ and the relaxed interfacial energy density $h$. Because our present interest lies in the case of disarrangement densities, and not on the full energetics of structured deformations, we shall restrict our attention to the case $W=0$, and we record the following adaptation for the case $W=0$ of results from [Choksi and Fonseca 1997] (see [Owen and Paroni 2015, Theorem 3] for further comments and other adaptations).

Theorem 1.1. Let $S^{N-1}=\left\{v \in \mathbb{R}^{N}:|v|=1\right\}$. Let $\Omega$ be a bounded open subset of $\mathbb{R}^{N}$ and assume $\psi: \mathbb{R}^{N} \times S^{N-1} \rightarrow[0,+\infty)$ satisfies the following conditions:

(H1) There exists a constant $C>0$ such that, for all $(\xi, v) \in \mathbb{R}^{N} \times S^{N-1}$,

$$
0 \leq \psi(\xi, \nu) \leq C|\xi|
$$

(H2) $\psi(\cdot, v)$ is positively homogeneous of degree 1: for all $t>0$ and $(\xi, v) \in$ $\mathbb{R}^{N} \times S^{N-1}$, we have

$$
\psi(t \xi, v)=t \psi(\xi, v)
$$

(H3) $\psi(\cdot, v)$ is subadditive; i.e., for all $\xi_{1}, \xi_{2} \in \mathbb{R}^{N}$ and $v \in S^{N-1}$,

$$
\psi\left(\xi_{1}+\xi_{2}, v\right) \leq \psi\left(\xi_{1}, v\right)+\psi\left(\xi_{2}, v\right)
$$

Then, for any $p>1$, if we define 


$$
\begin{aligned}
& I(g, G):=\inf \left\{\liminf _{n \rightarrow \infty} \int_{J\left(u_{n}\right) \cap \Omega} \psi\left(\left[u_{n}\right], v\right) d \mathcal{H}^{N-1}:\right. \\
& u_{n} \in S B V\left(\Omega ; \mathbb{R}^{N}\right), u_{n} \rightarrow g \text { in } L^{1}\left(\Omega ; \mathbb{R}^{N}\right), \\
&\left.\nabla u_{n} \stackrel{*}{\rightarrow} G, \sup _{n}\left(\left|\nabla u_{n}\right|_{L^{p}\left(\Omega ; \mathbb{R}^{N \times N}\right)}+\left|D u_{n}\right|(\Omega)\right)<+\infty\right\},
\end{aligned}
$$

we have

$$
I(g, G)=\int_{\Omega} H(\nabla g(x), G(x)) d \mathscr{L}^{N}+\int_{J(g) \cap \Omega} h([g](x), v(x)) d \mathscr{H}^{N-1}(x),
$$

where

$$
\begin{aligned}
H(A, B):=\inf \left\{\int_{J(u) \cap Q} \psi([u], v) d \mathscr{H}^{N-1}:\right. & \\
& u \in S B V\left(Q ; \mathbb{R}^{N}\right),\left.u\right|_{\partial Q}=A x, \\
& \left.|\nabla u| \in L^{p}(Q), \int_{Q} \nabla u d \mathscr{L}^{N}=B\right\}
\end{aligned}
$$

and

$$
\begin{aligned}
h(\xi, \eta):=\inf \left\{\int_{J(u) \cap Q_{\eta}} \psi([u], v) d \mathscr{H}^{N-1}:\right. \\
\left.u \in \operatorname{SBV}\left(Q_{\eta} ; \mathbb{R}^{N}\right),\left.u\right|_{\partial Q_{\eta}}=u_{\xi, \eta}, \nabla u=0 \text { a.e. }\right\}
\end{aligned}
$$

with

$$
u_{\xi, \eta}(x):= \begin{cases}0 & \text { if }-\frac{1}{2} \leq x \cdot \eta<0 \\ \xi & \text { if } \quad 0 \leq x \cdot \eta<\frac{1}{2}\end{cases}
$$

Here, $Q=\left(-\frac{1}{2}, \frac{1}{2}\right)^{N}$ and $Q_{\eta}$ denotes the unit cube centered at the origin and with two faces normal to $\eta$.

On the right side of (1-17), we have corrected an inconsequential misprint that is present in the corresponding formula in Theorem 3 of [Owen and Paroni 2015].

Another approach to relaxation of energies for structured deformations in the full $B V$ setting is provided in [Baía et al. 2012]. A structured deformation in [Baía et al. 2012] is a pair

where

$$
(g, G) \in B V^{2}\left(\Omega ; \mathbb{R}^{N}\right) \times B V\left(\Omega ; \mathbb{R}^{N \times N}\right),
$$

$$
B V^{2}\left(\Omega ; \mathbb{R}^{N}\right):=\left\{u \in B V\left(\Omega ; \mathbb{R}^{N}\right): \nabla u \in B V\left(\Omega ; \mathbb{R}^{N \times N}\right)\right\} .
$$

The counterpart of the approximation theorem in this context asserts that there exists a sequence $f_{n} \in B V^{2}\left(\Omega ; \mathbb{R}^{N}\right)$ such that both $f_{n} \rightarrow g$ and $\nabla f_{n} \rightarrow G$ in the $L^{1}$-norm. In this case, we write $f_{n} \rightsquigarrow(g, G)$. 
The energy functional considered in [Baía et al. 2012], under assumptions on the initial bulk and surface energy densities similar to the ones in [Choksi and Fonseca 1997], reads

$$
\begin{aligned}
E\left(f_{n}\right)=\int_{\Omega} W\left(\nabla f_{n}(y), \nabla^{2} f_{n}(y)\right) d \mathscr{L}^{N} y & +\int_{J\left(f_{n}\right)} \psi\left(\left[f_{n}\right](y), v(y)\right) d \mathscr{H}^{N-1}(y) \\
& +\int_{J\left(\nabla f_{n}\right)} \psi_{1}\left(\left[\nabla f_{n}\right](y), v(y)\right) d \mathscr{H}^{N-1}(y),
\end{aligned}
$$

and the relaxed energy $I(g, G)$ is defined by

$$
I(g, G):=\inf \left\{\liminf _{n \rightarrow \infty} E\left(f_{n}\right): f_{n} \rightsquigarrow(g, G)\right\} .
$$

A crucial result in [Baía et al. 2012] is that (1-19) can be divided into two first-order relaxed energies, namely, $I(g, G)=I_{1}(g, G)+I_{2}(G)$, where the term $I_{1}(g, G)$ captures the structured deformation whereas $I_{2}(G)$ only depends on the deformation without disarrangements $G$. In the relevant case for the present paper, i.e., $W=\psi_{1}=0$, the results in [Baía et al. 2012] give $I_{2}=0$ and

$$
I_{1}(g, G):=\inf \left\{\liminf _{n \rightarrow \infty} \int_{J\left(f_{n}\right) \cap \Omega} \psi\left(\left[f_{n}\right](y), v(y)\right) d \mathscr{H}^{N-1}(y): f_{n} \rightsquigarrow(g, G)\right\} .
$$

Defining $S B V^{2}\left(\Omega ; \mathbb{R}^{N}\right):=\left\{u \in S B V\left(\Omega ; \mathbb{R}^{N}\right): \nabla u \in S B V\left(\Omega ; \mathbb{R}^{N \times N}\right)\right\}$, the following representation theorem holds

Theorem 1.2 [Baía et al. 2012, Theorem 3.2]. For every $(g, G) \in S B V^{2}\left(\Omega ; \mathbb{R}^{N}\right) \times$ $\operatorname{SBV}\left(\Omega ; \mathbb{R}^{N \times N}\right)$, given $\psi$ under the same hypotheses $(\mathrm{H} 1)-(\mathrm{H} 3)$ of Theorem 1.1,

$$
I(g, G)=\int_{\Omega} H(G(x)-\nabla g(x)) d \mathscr{L}^{N}+\int_{J(g) \cap \Omega} h([g](x), v(x)) d \mathscr{H}^{N-1}(x),
$$

where, given $A \in \mathbb{R}^{N \times N}, \xi \in \mathbb{R}^{N}$, and $\eta \in S^{N-1}$,

$$
\begin{aligned}
& H(A):=\inf \left\{\int_{J(u) \cap Q} \psi([u], v) d \mathscr{H}^{N-1}:\right. \\
& \left.u \in S B V^{2}\left(Q ; \mathbb{R}^{N}\right),\left.u\right|_{\partial Q}=0, \nabla u=A \text { a.e. in } Q\right\}
\end{aligned}
$$

and

$$
\begin{aligned}
h(\xi, \eta):=\inf \left\{\int_{J(u) \cap Q_{\eta}} \psi([u], v) d \mathcal{H}^{N-1}:\right. \\
\left.u \in S B V^{2}\left(Q_{\eta} ; \mathbb{R}^{N}\right),\left.u\right|_{\partial Q_{\eta}}=u_{\xi, \eta}, \nabla u=0 \text { a.e. in } Q\right\},
\end{aligned}
$$

with $u_{\xi, \eta}$ defined as in (1-18). 
Remark 1.3. It is worth noticing that the minimum problems defining (1-20) and (1-21) are formally performed in $S B V^{2}\left(\Omega ; \mathbb{R}^{N}\right)$, but the result is the same if $S B V^{2}$ is replaced in these relations by $S B V$, due to the requirement that $\nabla u$ be constant.

1D. Explicit formulas for relaxed disarrangement densities. Owen and Paroni [2015] applied Theorem 1.1 to the specific disarrangement densities $\left|\left[f_{n}\right](y) \cdot v(y)\right|$ and $\left(\left[f_{n}\right](y) \cdot v(y)\right)^{ \pm}$introduced in Section 1A and obtained for each of these densities an explicit formula for the corresponding relaxed disarrangement densities $H$ in (1-16) and $h$ in (1-17). Among their results [Owen and Paroni 2015, Theorem 4] is the following (obtained by setting $L(x)=I$ in their Theorem 4):

Theorem 1.4. The initial disarrangement densities

$$
\begin{aligned}
\psi^{|\cdot|}(\xi, v) & :=|\xi \cdot v|, \\
\psi^{ \pm}(\xi, v) & :=(\xi \cdot v)^{ \pm}
\end{aligned}
$$

satisfy the hypotheses $(\mathrm{H1})-(\mathrm{H} 3)$ in Theorem 1.1 and have relaxed disarrangement densities given by

$$
\begin{aligned}
H^{|\cdot|}(A, B) & =|\operatorname{tr}(A-B)|, & h^{|\cdot|}(\xi, v) & =|\xi \cdot v|=\psi^{|\cdot|}(\xi, v), \\
H^{ \pm}(A, B) & =(\operatorname{tr}(A-B))^{ \pm}, & h^{ \pm}(\xi, v) & =(\xi \cdot v)^{ \pm}=\psi^{ \pm}(\xi, v) .
\end{aligned}
$$

Specifically, when the minimal volume that is swept out by disarrangements $\mathscr{V}^{|\cdot|}(g, G ; \mathscr{P})$ is defined in the Choksi-Fonseca setting by (1-11), then (1-23) yields the explicit formula

$$
\begin{aligned}
& \mathscr{V}^{|\cdot|}(g, G ; \mathscr{P}) \\
& \quad=\int_{\mathscr{P}}|\operatorname{tr}(\nabla g(x)-G(x))| d \mathscr{L}^{N}(x)+\int_{J(g) \cap \mathscr{P}}|[g](x) \cdot v(x)| d \mathscr{H}^{N-1}(x)
\end{aligned}
$$

for the (minimal) volume swept out by separations and switches among approximations $f_{n}$ that determine $(g, G)$. Relation (1-24) provides answers in the setting of Choksi and Fonseca to the questions raised at the end of Section 1A: ${ }^{\mathscr{V}}|\cdot|(g, G ; \mathscr{P})$ has both a bulk disarrangement density $|\operatorname{tr}(\nabla g-G)|=|\operatorname{tr} M|$ and an interfacial disarrangement density $|[g] \cdot v|$. Similarly, Theorem 1.4 shows that the (minimal) volume swept out by separations alone, $\mathscr{V}^{+}(g, G ; \mathscr{P})$, has the bulk disarrangement density $(\operatorname{tr} M)^{+}$and the interfacial disarrangement density $([g] \cdot v)^{+}$, with a corresponding result for $\mathscr{V}^{-}(g, G ; \mathscr{P})$, the (minimal) volume swept out by switches and interpenetrations (the approximations $f_{n}$ in the Choksi-Fonseca setting are not required to be injective so that interpenetrations can arise there, unlike in the setting of Del Piero-Owen). 
1E. Summary of the research presented in the present article. In the proof of Theorem 1.4 given in [Owen and Paroni 2015], the significant part of the argument addresses the verification of the inequality

$$
H^{|\cdot|}(A, B) \leq|\operatorname{tr}(A-B)|,
$$

where $H^{|\cdot|}(A, B)$ is given by the right-hand side of (1-16) with $\psi\left([u], v_{u}\right)$ replaced by $\psi^{|\cdot|}([u], v)=|[u] \cdot v|$. This inequality was proved in [Owen and Paroni 2015] by constructing a family $u_{\varepsilon}$ of piecewise affine mappings on the unit cube $Q$, each of whose jump set $J\left(u_{\varepsilon}\right)$ is formed by two (planar) ends and by a lateral surface constructed from solution curves of the differential equation $\dot{x}=(A-B) x$. The lateral surface, by construction, contributes nothing to $\int_{J(u) \cap Q_{\eta}}|[u] \cdot v| d \mathscr{H}^{N-1}$, and the contributions of the two ends can be calculated explicitly for $A-B$ lying in a dense subset of $\mathbb{R}^{N \times N}$. Proposition 5.2 of [Choksi and Fonseca 1997] provides sufficient regularity of $H^{|\cdot|}(A, B)$ to establish (1-25) for all $A-B \in \mathbb{R}^{N \times N}$.

As one of the main results in this article, we provide an alternate, shorter proof of (1-25) that employs a different family $u_{\varepsilon}$ of piecewise affine mappings that does not involve solution curves of $\dot{x}=(A-B) x$. Our approach is based on the following observation. With $A, B \in \mathbb{R}^{N \times N}, p>1$, and $Q=\left(-\frac{1}{2}, \frac{1}{2}\right)^{N}$,

$$
\begin{gathered}
|\operatorname{tr}(A-B)| \leq \inf \left\{\int_{J(u)}|[u](x) \cdot v(x)| d \mathscr{H}^{N-1}(x): u \in S B V\left(Q ; \mathbb{R}^{N}\right),\right. \\
\left.u(x)=A x \text { on } \partial Q, \nabla u \in L^{p}(Q), \int_{Q} \nabla u(x) d \mathscr{L}^{N}(x)=B\right\} \\
\leq \inf \left\{\int_{J(u)}|[u](x) \cdot v(x)| d \mathscr{H}^{N-1}(x): u \in S B V\left(Q ; \mathbb{R}^{N}\right),\right. \\
u(x)=0 \text { on } \partial Q, \nabla u=B-A \text { a.e. }\} .
\end{gathered}
$$

The first inequality follows by moving the absolute value outside the integral and using the Gauss-Green theorem for the space $S B V\left(Q ; \mathbb{R}^{N}\right)$ of special functions of bounded variation while the second follows by noting that, if $u$ satisfies the last set of conditions, then the function $x \mapsto u(x)+A x$ satisfies the first set of conditions. In this paper, we wish to show that

$$
\begin{aligned}
\inf \left\{\int_{J(u)}|[u](x) \cdot v(x)| d \mathscr{H}^{N-1}(x): u \in S B V\left(Q ; \mathbb{R}^{N}\right),\right. \\
u(x)=0 \text { on } \partial Q, \nabla u=B-A \text { a.e. }\} \leq|\operatorname{tr}(A-B)|
\end{aligned}
$$

so that the two infima in (1-26) have common value $|\operatorname{tr}(A-B)|$.

The second main contribution of the present research concerns the alternative approach to structured deformations and to relaxed energies due to Baía, Matias, 
and Santos [Baía et al. 2012] discussed at the end of Section 1C. According to that discussion, the second infimum in (1-26) (see (1-20) and Remark 1.3)

$$
\begin{aligned}
& \inf \left\{\int_{J(u)}|[u](x) \cdot v(x)| d \mathscr{H}^{N-1}(x):\right. \\
& \left.\qquad u \in \operatorname{SBV}\left(Q ; R^{N}\right), u(x)=0 \text { on } \partial Q, \nabla u=B-A \text { a.e. in } Q\right\}
\end{aligned}
$$

is the bulk disarrangement density for the same interfacial disarrangement density $\psi^{|\cdot|}([u], v)(1-22)$ studied by Owen and Paroni in the setting of Choksi and Fonseca. Consequently, our proof of (1-27) establishes the equality of the bulk disarrangement densities obtained in two different settings for structured deformations. Thus, the geometrical significance of the expression $|\operatorname{tr}(A-B)|$ described in [Owen and Paroni 2015], namely, a volume density of volume swept out by nonsmooth, submacroscopic geometrical changes, is strengthened by the fact that the same expression arises from two different schemes of relaxation. We note that the two different schemes of relaxation also deliver the same formula for the (relaxed) interfacial disarrangement density $h: h=\psi^{|\cdot|}$ (see [Owen and Paroni 2015] for the routine verification that applies to both schemes).

The explicit formulas for disarrangement densities considered here in the context of structured deformations will provide scalar fields that can enter as variables in constitutive relations for the response of three-dimensional bodies. For this purpose, frame-indifferent variants of the specific fields obtained here are available through known factorizations of structured deformations in which the factor that tracks disarrangements is unchanged under changes in frame [Del Piero and Owen 1993]. Our explicit formulas are also starting points for the study of examples in other contexts involving structured deformations: second-order structured deformations [Owen and Paroni 2000] in which second gradients and their limits enter into submacroscopic changes in geometry as well as processes for dimension reduction [Matias and Santos 2014] in the presence of disarrangements that describe thin structures undergoing submacroscopic slips, separations, and switches.

In Section 2, we provide a "tilted cube" construction for the family $u_{\varepsilon}$ of functions employed in proving (1-27). The common orientation of the tilted cubes is determined in Section 3 by means of a known result on the isotropic vectors of symmetric linear mappings. The proof of (1-27) is completed in Section 4, and the paper concludes with Section 5 with some additional explicit formulas for disarrangement densities.

During the review of this article, the research of [Šilhavý 2016] was brought to our attention, in which explicit formulas for the bulk and interfacial relaxed energies are established for a broad class of purely interfacial initial energies that includes the ones studied here. 


\section{Proof of the upper-bound inequality}

In what follows, a proof of (1-27) is given. The proof requires the following instance of Lemma 4.3 in [Matias 2007].

Lemma 2.1. Let $M \in \mathbb{R}^{N \times N}$ and a bounded open set $\Omega \subset \mathbb{R}^{N}$ be given, with $\Omega$ having Lipshitz boundary. There exist a number $C(N)>0$, independent of $M$ and $\Omega$, and $u \in \operatorname{SBV}\left(\Omega ; \mathbb{R}^{N}\right)$ such that

(1) $\left.u\right|_{\partial \Omega}=0$,

(2) $\nabla u=M, \mathscr{L}^{N}$-a.e. on $\Omega$, and

(3) $\left|D^{s} u\right|(\Omega) \leq C(N)\|M\| \mathscr{L}^{N}(\Omega)$.

Here, $\nabla u$ and $D^{s} u$ denote the absolutely continuous and the singular parts of the distributional derivative $D u=\nabla u \mathscr{L}^{N}+D^{s} u$ of $u$, and $\left|D^{s} u\right|$ denotes the total variation of the singular part. In addition, $\|M\|:=\left(\operatorname{tr}\left(M^{T} M\right)\right)^{1 / 2}$ is the Euclidean norm of the matrix $M$. We shall now use the lemma to verify (1-27) for $M=A-B$. To this end, let an integer $n \geq 1$ be given and consider the frame

$$
\mathscr{F}_{n}:=Q \backslash \overline{\left(1-\frac{2}{n+2}\right) Q} \text {. }
$$

We may apply the lemma to obtain an $S B V$ function $u^{(n)}: \mathscr{F}_{n} \rightarrow \mathbb{R}^{N}$ such that

- $\left.u^{(n)}\right|_{\partial \mathscr{F}_{n}}=0$,

- $\nabla u^{(n)}=M, \mathscr{L}^{N}$-a.e. on $\mathscr{F}_{n}$, and

- the total variation $\int_{J\left(u^{(n)}\right)}\left|\left[u^{(n)}\right]\right|(x) d \mathscr{H}^{N-1}(x)$ of $u^{(n)}$ satisfies

$$
\int_{J\left(u^{(n)}\right)}\left|\left[u^{(n)}\right]\right|(x) d \mathscr{H}^{N-1}(x) \leq C(N)\|M\|\left(1-\left(1-\frac{2}{n+2}\right)^{N}\right) .
$$

In preparation for defining an appropriate function $u$ on $Q \backslash \overline{\mathscr{F}_{n}}=\left(1-\frac{2}{n+2}\right) Q$, we write $\widehat{M}:=\frac{1}{2}\left(M+M^{T}\right)$ for the symmetric part of $M$, and we choose an orthonormal basis $e_{i}, i=1, \ldots, N$, of $\mathbb{R}^{N}$ that consists of eigenvectors of $\widehat{M}$ :

$$
\widehat{M} e_{i}=\lambda_{i} e_{i}, \quad i=1, \ldots, N .
$$

We let $m$ be a positive integer and cover $\left(1-\frac{2}{n+2}\right) Q$ by a collection $\mathscr{C}_{n, m}$ of congruent, nonoverlapping open cubes $C_{n, m}^{k}, k=1, \ldots, K_{n, m}$, each of edge-length $1 / m$ and each with the $i$-th pair of opposite faces orthogonal to the unit vector $R e_{i}$, for $i=1, \ldots, N$. Here, $R$ is an orthogonal $N \times N$ matrix, $R R^{T}=R^{T} R=I$, to be determined presently. We require in addition that each cube $C_{n, m}^{k}$ satisfies

$$
\left(1-\frac{2}{n+2}\right) Q \cap C_{n, m}^{k} \neq \varnothing \text {. }
$$


We denote by $c_{n, m}^{k}$ the center of $C_{n, m}^{k}$, and we define $u_{n, m}:\left(1-\frac{2}{n+2}\right) Q \rightarrow \mathbb{R}^{N}$ by $u_{n, m}(x):= \begin{cases}M\left(x-c_{n, m}^{k}\right) & \text { if } x \in\left(1-\frac{2}{n+2}\right) Q \cap C_{n, m}^{k} \text { for some } k=1, \ldots, K_{n, m}, \\ 0 & \text { if } x \in\left(1-\frac{2}{n+2}\right) Q \backslash \bigcup_{k=1}^{K_{n, m}} C_{n, m}^{k} .\end{cases}$

Using standard reasoning, we conclude that $u_{n, m} \in S B V\left(\left(1-\frac{2}{n+2}\right) Q ; \mathbb{R}^{N}\right)$ with $\nabla u_{n, m}=M, \mathscr{L}^{N}$-a.e. on $\left(1-\frac{2}{n+2}\right) Q$. Moreover, the trace of $u_{n, m}$ on $\partial\left(\left(1-\frac{2}{n+2}\right) Q\right)$ is bounded pointwise by $(\sqrt{N} / 2 m)\|M\|$. Consequently, the function $u_{m}^{(n)}: Q \rightarrow \mathbb{R}^{N}$ defined by

$$
u_{m}^{(n)}(x):= \begin{cases}u^{(n)}(x) & \text { for } x \in \mathscr{F}_{n}, \\ u_{n, m}(x) & \text { for } x \in\left(1-\frac{2}{n+2}\right) Q\end{cases}
$$

belongs to $\operatorname{SBV}\left(Q ; \mathbb{R}^{N}\right)$, has gradient $M, \mathscr{L}^{N}$-a.e., and has zero trace on $\partial Q$. Moreover, the jump set of $u_{m}^{(n)}$ satisfies

$$
J\left(u_{m}^{(n)}\right) \subset J\left(u^{(n)}\right) \cup \partial\left(\left(1-\frac{2}{n+2}\right) Q\right) \cup J\left(u_{n, m}\right) .
$$

Since $u_{m}^{(n)}$ has outer trace 0 on $\partial\left(1-\frac{2}{n+2}\right) Q$, for $\mathscr{H}^{N-1}$-a.e. $x$ in $\partial\left(\left(1-\frac{2}{n+2}\right) Q\right)$

$$
\left|\left[u_{m}^{(n)}\right](x)\right| \leq \frac{\sqrt{N}}{m}\|M\|
$$

and, consequently,

$$
\int_{\partial((1-2 /(n+2)) Q)}\left|\left[u_{m}^{(n)}\right](x) \cdot v(x)\right| d \mathscr{H}^{N-1}(x) \leq \frac{\sqrt{N}}{m}\|M\| 2 N\left(1-\frac{2}{n+2}\right)^{N-1} .
$$

We note from (2-1) that

$$
\int_{J\left(u^{(n)}\right)}\left|\left[u_{m}^{(n)}\right](x) \cdot v(x)\right| d \mathscr{H}^{N-1}(x) \leq C(N)\|M\|\left(1-\left(1-\frac{2}{n+2}\right)^{N}\right)
$$

and we seek a corresponding estimate for $\int_{J\left(u_{n, m}\right)}\left|\left[u_{m}^{(n)}\right](x) \cdot v(x)\right| d \mathscr{H}^{N-1}(x)$. To this end, we note that

$$
J\left(u_{n, m}\right) \subset \bigcup_{k=1}^{K_{n, m}} \partial C_{n, m}^{k},
$$

and we shall seek an upper bound for $\int_{\bigcup_{k=1}^{K n, m} \partial C_{n, m}^{k}}\left|\left[u_{m}^{(n)}\right](x) \cdot v(x)\right| d \mathscr{H}^{N-1}(x)$. For each $k=1, \ldots, K_{n, m}$ and $i=1, \ldots, N$, we denote by $\phi_{n, m}^{k, i+}$ and $\phi_{n, m}^{k, i-}$ the two faces of the cube $C_{n, m}^{k} \in \mathscr{C}_{n, m}$ orthogonal to $R e_{i}$. We note that one face $\phi_{n, m}^{k, i+}$ of $C_{n, m}^{k}$ has outer normal $v_{i, k}^{+}=+R e_{i}$ while the opposite face $\phi_{n, m}^{k, i-}$ has outer normal $v_{i, k}^{-}=-R e_{i}$.

We suppose now that the face $\phi_{n, m}^{k, i+}$ of $C_{n, m}^{k} \in \mathscr{C}_{n, m}$ satisfies

$$
\phi_{n, m}^{k, i+} \subset\left(1-\frac{2}{n+2}\right) Q \text {. }
$$


Then there is a cube $C_{n, m}^{k^{\prime}} \in \mathscr{C}_{n, m}$ that shares the given face with $C_{n, m}^{k}$, and we have at each point $x \in \phi_{n, m}^{k, i+}$ the equality

$$
\begin{aligned}
{\left[u_{m}^{(n)}\right](x) \cdot v(x) } & =\left(M\left(x-c_{n, m}^{k^{\prime}}\right)-M\left(x-c_{n, m}^{k}\right)\right) \cdot v_{i, k}^{+}(x) \\
& =M\left(c_{n, m}^{k}-c_{n, m}^{k^{\prime}}\right) \cdot v_{i, k}^{+}(x) \\
& =M\left(-\frac{1}{m} v_{i, k}^{+}(x)\right) \cdot v_{i, k}^{+}(x)=-\frac{1}{m} \widehat{M} \operatorname{Re}_{i} \cdot R e_{i}
\end{aligned}
$$

so that

$$
\begin{aligned}
\int_{\phi_{n, m}^{k, i+}}\left|\left[u_{m}^{(n)}\right](x) \cdot v(x)\right| d \mathcal{H}^{N-1}(x) & =\int_{\phi_{n, m}^{k, i+}} \frac{1}{m}\left|\widehat{M} R e_{i} \cdot \operatorname{Re}_{i}\right| d \mathcal{H}^{N-1}(x) \\
& =\frac{1}{m^{N}}\left|\widehat{M} R e_{i} \cdot R e_{i}\right| .
\end{aligned}
$$

The same argument shows that if

$$
\phi_{n, m}^{k, i-} \subset\left(1-\frac{2}{n+2}\right) Q
$$

then

$$
\int_{\phi_{n, m}^{k, i-}}\left|\left[u_{m}^{(n)}\right](x) \cdot v(x)\right| d \mathscr{H}^{N-1}(x)=\frac{1}{m^{N}}\left|\widehat{M} R e_{i} \cdot R e_{i}\right| .
$$

If (2-5) holds for $i=1, \ldots, N$, then we may sum the last relation over $i$ to conclude that

$$
\begin{aligned}
\sum_{i=1}^{N} \int_{\phi_{n, m}^{k, i}}\left|\left[u_{m}^{(n)}\right](x) \cdot v(x)\right| d \mathscr{H}^{N-1}(x) & =\frac{1}{m^{N}} \sum_{i=1}^{N}\left|\widehat{M} R e_{i} \cdot R e_{i}\right| \\
& \geq \frac{1}{m^{N}}\left|\sum_{i=1}^{N} \widehat{M} R e_{i} \cdot R e_{i}\right| \\
& =\frac{1}{m^{N}}\left|\sum_{i=1}^{N} R^{T} \widehat{M} R e_{i} \cdot e_{i}\right| \\
& =\frac{1}{m^{N}}\left|\operatorname{tr}\left(R^{T} \widehat{M} R\right)\right|=\frac{1}{m^{N}}|\operatorname{tr} M| .
\end{aligned}
$$

In (2-8), equality holds if and only if all of the numbers $\widehat{M} R e_{i} \cdot R e_{i}, i=1, \ldots, N$, have the same sign:

$$
\left(\widehat{M} R e_{i} \cdot R e_{i}\right)\left(\widehat{M} R e_{j} \cdot R e_{j}\right) \geq 0 \text { for } i, j=1, \ldots, N .
$$

The last two inequalities lead us to consider the problem

$$
\text { find } \min _{R R^{T}=I} \sum_{i=1}^{N}\left|\widehat{M} R e_{i} \cdot R e_{i}\right| \geq|\operatorname{tr} \widehat{M}|=|\operatorname{tr} M|,
$$

with equality holding if and only if there is an orthogonal matrix $R$ satisfying (2-9). 


\section{Aside on isotropic vectors}

We note that the sign inequality (2-9) suggests looking for unit vectors $v$ such that

$$
\widehat{M} v \cdot v=0,
$$

the isotropic vectors for $\widehat{M}$ [Ciblak and Lipkin 1998]. In particular, in the special case $\operatorname{tr} \widehat{M}=0$, the existence of $N$ mutually orthogonal isotropic vectors $v_{1}, \ldots, v_{N}$ would ensure that the matrix $R$ defined by $R e_{i}=v_{i}$ for $i=1, \ldots, N$ would satisfy (2-10) in the form $0=0$. More generally, even when $\operatorname{tr} \widehat{M} \neq 0$, the existence of isotropic vectors is useful. In fact, the symmetric matrix $\widehat{M}-\frac{1}{N}(\operatorname{tr} \widehat{M}) I$ has zero trace, so we suppose that there exist $N$ mutually orthogonal isotropic unit vectors $v_{1}, \ldots, v_{N}$ for $\widehat{M}-\frac{1}{N}(\operatorname{tr} \widehat{M}) I$. The relation (3-1) with $\widehat{M}$ replaced by $\widehat{M}-\frac{1}{N}(\operatorname{tr} \widehat{M}) I$ then becomes

$$
0=\left(\widehat{M}-\frac{1}{N}(\operatorname{tr} \widehat{M}) I\right) v_{i} \cdot v_{i}=\widehat{M} v_{i} \cdot v_{i}-\frac{1}{N} \operatorname{tr} \widehat{M}
$$

so that $\widehat{M} v_{i} \cdot v_{i}=\frac{1}{N} \operatorname{tr} \widehat{M}$ for $i=1, \ldots, N$. Again, if we define a linear mapping $R$ on $\mathbb{R}^{N}$ by $R e_{i}=v_{i}$ for $i=1, \ldots, N$, then $R$ is orthogonal, it satisfies the sign inequality for $\widehat{M}$ (2-9), and it delivers equality in (2-10) in the form $\sum_{i=1}^{N}\left|\frac{1}{N} \operatorname{tr} \widehat{M}\right|=|\operatorname{tr} \widehat{M}|$.

The following result [Ciblak and Lipkin 1998, Corollary 15] provides the desired existence of complete orthonormal sets of isotropic vectors.

Theorem 3.1. A symmetric matrix $A \in \mathbb{R}^{N \times N}$ possesses an orthonormal set of $N$ isotropic vectors if and only if $\operatorname{tr} A=0$.

This theorem and the preceding discussion permit us to conclude: for every matrix $M \in \mathbb{R}^{N \times N}$,

$$
\begin{aligned}
\min _{R R^{T}=I} \sum_{i=1}^{N}\left|M R e_{i} \cdot R e_{i}\right| & =\min _{R R^{T}=I} \sum_{i=1}^{N}\left|\widehat{M} R e_{i} \cdot R e_{i}\right| \\
& =|\operatorname{tr} \widehat{M}|=|\operatorname{tr} M|,
\end{aligned}
$$

and a minimizing rotation matrix $R$ is one carrying the orthonormal basis of $\mathbb{R}^{N}$ consisting of eigenvectors of $\widehat{M}$ into an orthonormal basis of $\mathbb{R}^{N}$ consisting of isotropic vectors of $\widehat{M}-\frac{1}{N}(\operatorname{tr} \widehat{M}) I$. For this minimizing rotation matrix, we have

$$
\left|\widehat{M} R e_{i} \cdot R e_{i}\right|=\frac{1}{N}|\operatorname{tr} M| \quad \text { for } i=1, \ldots, N .
$$

We remark that minimizers are not unique, in general, even when one eliminates trivial permutations of isotropic vectors. In fact, for $N=3$, there are examples of minimizers for which two of the three terms in $\sum_{i=1}^{3}\left|\widehat{M} R e_{i} \cdot R e_{i}\right|$ vanish, while the third equals $|\operatorname{tr} M|$, so that only two of the three vectors $R e_{i}$ are isotropic vectors for $\widehat{M}$. 
For the convenience of the reader, we provide the recursive step used in proving the existence of orthonormal bases made up of isotropic vectors for a traceless symmetric matrix $A \in \mathbb{R}^{N \times N}$. We interpret $A$ in the usual way as a linear mapping on $\mathbb{R}^{N}$, endowed with the standard inner product. Then the nullspace $\operatorname{Ker} A$ of $A$ and its orthogonal complement $(\operatorname{Ker} A)^{\perp}$ are complementary $A$-invariant subspaces of $\mathbb{R}^{N}$, and all vectors in $\operatorname{Ker} A$ are isotropic vectors for $A$. If $(\operatorname{Ker} A)^{\perp}$ is the zero subspace, then $A=0$ and every vector in $\mathbb{R}^{N}$ is an isotropic vector for $A$, and every orthonormal basis of $\mathbb{R}^{N}$ meets the desired requirement. If $(\operatorname{Ker} A)^{\perp}$ is not the zero subspace, then we seek additional isotropic vectors for $A$ in $(\operatorname{Ker} A)^{\perp}$. To this end, the traceless symmetric linear mapping $A \neq 0$ has both positive and negative eigenvalues so that

$$
\min _{|u|=1} A u \cdot u<0<\max _{|u|=1} A u \cdot u
$$

and since the unit sphere in $\mathbb{R}^{N}$ is connected and since the quadratic form $u \mapsto A u \cdot u$ is continuous, there exists a unit vector $v_{1} \in \mathbb{R}^{N}$ such that $A v_{1} \cdot v_{1}=0$. Writing $v_{1}$ as a sum of two orthogonal vectors, one in $\operatorname{Ker} A$ and the other in $(\operatorname{Ker} A)^{\perp}$, and using the invariance of $(\operatorname{Ker} A)^{\perp}$ under $A$ shows that we may without loss of generality assume that $v_{1} \in(\operatorname{Ker} A)^{\perp}$. The linear $\operatorname{span} \operatorname{Lsp}\left(\operatorname{Ker} A \cup\left\{v_{1}\right\}\right)$ has dimension one larger than that of $\operatorname{Ker} A$ and consists solely of isotropic vectors for $A$. Consequently, we need to search for isotropic vectors of $A$ in $\left(\operatorname{Lsp}\left(\operatorname{Ker} A \cup\left\{v_{1}\right\}\right)\right)^{\perp}$ which has dimension one less than $(\operatorname{Ker} A)^{\perp}$. To proceed further, we define a linear mapping $A_{1}$ on $\mathbb{R}^{N}$ by

$$
A_{1}=A-v_{1} \otimes A v_{1}-A v_{1} \otimes v_{1}
$$

where the formula $(a \otimes b) v:=(b \cdot v) a$, for all $a, b, v \in \mathbb{R}^{N}$, defines the standard tensor product $a \otimes b \in \operatorname{Lin}\left(\mathbb{R}^{N} ; \mathbb{R}^{N}\right)$. From the fact that $v_{1}$ is an isotropic vector for $A$ and from the formula $\operatorname{tr}(a \otimes b)=a \cdot b$, it is easy to see that $A_{1}$ is traceless; because $(a \otimes b)^{T}=b \otimes a$, it follows that $A_{1}$ is symmetric. In addition, if $v \in$ $\left(\operatorname{Lsp}\left(\operatorname{Ker} A \cup\left\{v_{1}\right\}\right)\right)^{\perp}$ is an isotropic vector for $A_{1}$, then we not only have $v \cdot v_{1}=0$ but also

$$
\begin{aligned}
0 & =A_{1} v \cdot v \\
& =\left(A v-\left(A v_{1} \cdot v\right) v_{1}-\left(v_{1} \cdot v\right) A v_{1}\right) \cdot v \\
& =A v \cdot v-\left(A v_{1} \cdot v\right)\left(v_{1} \cdot v\right)-\left(v_{1} \cdot v\right)\left(A v_{1} \cdot v\right) \\
& =A v \cdot v
\end{aligned}
$$

Thus, every isotropic vector for $A_{1}$ that is in $\left(\operatorname{Lsp}\left(\operatorname{Ker} A \cup\left\{v_{1}\right\}\right)\right)^{\perp}$ is an isotropic vector for $A$, and $\operatorname{dim}\left(\left(\operatorname{Lsp}\left(\operatorname{Ker} A \cup\left\{v_{1}\right\}\right)\right)^{\perp}\right)=\operatorname{dim}\left((\operatorname{Ker} A)^{\perp}\right)-1$. To be able to apply the foregoing considerations to $A_{1}$, we need only show that $\left(\operatorname{Lsp}\left(\operatorname{Ker} A \cup\left\{v_{1}\right\}\right)\right)^{\perp}$ is invariant under $A_{1}$. To this end, let $v \in\left(\operatorname{Lsp}\left(\operatorname{Ker} A \cup\left\{v_{1}\right\}\right)\right)^{\perp}, v_{\kappa} \in \operatorname{Ker} A$, and 
$\alpha \in \mathbb{R}$ be given, and consider

$$
\begin{aligned}
A_{1} v \cdot\left(v_{\kappa}+\alpha v_{1}\right) & =A_{1} v \cdot v_{\kappa}+A_{1} v \cdot \alpha v_{1} \\
& =v \cdot A_{1} v_{\kappa}+\alpha v \cdot A_{1} v_{1} \\
& =0+\alpha v \cdot\left(A v_{1}-\left(A v_{1} \otimes v_{1}\right) v_{1}-\left(v_{1} \otimes A v_{1}\right) v_{1}\right) \\
& =\alpha v \cdot\left(A v_{1}-\left(v_{1} \cdot v_{1}\right) A v_{1}-\left(A v_{1} \cdot v_{1}\right) v_{1}\right) \\
& =\alpha v \cdot\left(A v_{1}-A v_{1}-0\right)=0 .
\end{aligned}
$$

We may conclude that $A_{1} v \in\left(\operatorname{Lsp}\left(\operatorname{Ker} A \cup\left\{v_{1}\right\}\right)\right)^{\perp}$ as desired. In the third line of the above computation, we have used the side calculation

$$
\begin{aligned}
v \cdot A_{1} v_{\kappa} & =v \cdot\left(A-v_{1} \otimes A v_{1}-A v_{1} \otimes v_{1}\right) v_{\kappa} \\
& =v \cdot A v_{\kappa}-\left(A v_{1} \cdot v_{\kappa}\right)\left(v \cdot v_{1}\right)-\left(v_{1} \cdot v_{\kappa}\right)\left(v \cdot A v_{1}\right)=0
\end{aligned}
$$

The first term on the last line vanishes because $v_{\kappa} \in \operatorname{Ker} A$, the second vanishes because $v \in\left(\operatorname{Lsp}\left(\operatorname{Ker} A \cup\left\{v_{1}\right\}\right)\right)^{\perp}$, and the third vanishes because $v_{1} \in(\operatorname{Ker} A)^{\perp}$. The search for isotropic vectors for $A$ on the $A$-invariant subspace $(\operatorname{Ker} A)^{\perp}$ may now be replaced by the search for isotropic vectors for $A_{1}$ on the $A_{1}$-invariant subspace $\left(\operatorname{Lsp}\left(\operatorname{Ker} A \cup\left\{v_{1}\right\}\right)\right)^{\perp}$ of dimension one less than that of $(\operatorname{Ker} A)^{\perp}$.

\section{Completion of the proof of the upper-bound inequality}

We may use (3-2) and the formulas (2-6) and (2-7) to conclude: if $C_{n, m}^{k}$ has a face $\phi_{n, m}^{k, i \pm} \subset\left(1-\frac{2}{N+2}\right) Q$, then

$$
\int_{\phi_{n, m}^{k, i \pm}}\left|\left[u_{m}^{(n)}\right](x) \cdot v(x)\right| d \mathscr{H}^{N-1}(x)=\frac{|\operatorname{tr} M|}{N m^{N}}=\frac{|\operatorname{tr} M|}{N} \mathscr{L}^{N}\left(C_{n, m}^{k}\right) .
$$

On the other hand, if a face $\phi_{n, m}^{k, i \pm}$ of $C_{n, m}^{k} \in \mathscr{C}_{n, m}$ fails to satisfy $\phi_{n, m}^{k, i \pm} \subset\left(1-\frac{2}{N+2}\right) Q$, then the argument used to verify (4-1) may be applied to $\phi_{n, m}^{k, i \pm} \cap\left(1-\frac{2}{n+2}\right) Q$ to conclude that

$$
\int_{\phi_{n, m}^{k, i \pm} \cap(1-2 /(n+2)) Q}\left|\left[u_{m}^{(n)}\right](x) \cdot v(x)\right| d \mathscr{H}^{N-1}(x) \leq \frac{|\operatorname{tr} M|}{N} \mathscr{L}^{N}\left(C_{n, m}^{k}\right) .
$$

We now consider the cube $C_{n, m}^{1} \in \mathscr{C}_{n, m}$ and choose $V_{n, m}^{1}$, one of its $2^{N}$ vertices. Exactly $N$ faces $\phi^{1, j}, j=1, \ldots, N$, of $C_{n, m}^{1}$ meet at $V_{n, m}^{1}$. Because each cube $C_{n, m}^{k} \in \mathscr{C}_{n, m}$ for $k=1, \ldots, K_{n, m}$ can be obtained from $C_{n, m}^{1}$ by a unique translation $T_{k}$, the choices $C_{n, m}^{1}$ and $V_{n, m}^{1}$ induce via $T_{k}$ an assignment of $N$ faces $\phi^{k, j}$, $j=1, \ldots, N$, to $C_{n, m}^{k}$. It is easy to show that for all $k, k^{\prime}=1, \ldots, K_{n, m}$

$$
k^{\prime} \neq k \Longrightarrow\left\{\phi^{k^{\prime}, j}: j=1, \ldots, N\right\} \cap\left\{\phi^{k, j}: j=1, \ldots, N\right\}=\varnothing,
$$


i.e., the set of $N$ faces assigned to different cubes are disjoint. If we now apply the mapping

$$
C_{n, m}^{k} \mapsto\left\{\phi^{k, j}: j=1, \ldots, N\right\}
$$

to each cube in the collection

$$
\mathscr{C}_{n, m}^{\mathrm{int}}:=\left\{C_{n, m}^{k} \in \mathscr{C}_{n, m}: C_{n, m}^{k} \subset\left(1-\frac{2}{N+2}\right) Q\right\}
$$

then all of the faces $\phi^{k, j}$ so obtained will be included in $\left(1-\frac{2}{N+2}\right) Q$, and we may apply (4-1) to each such face to obtain for each $C_{n, m}^{k} \in \mathscr{C}_{n, m}^{\text {int }}$

$$
\sum_{j=1}^{N} \int_{\phi^{k, j}}\left|\left[u_{m}^{(n)}\right](x) \cdot v(x)\right| d \mathscr{H}^{N-1}(x)=N \frac{|\operatorname{tr} M|}{N} \mathscr{L}^{N}\left(C_{n, m}^{k}\right)=|\operatorname{tr} M| \mathscr{L}^{N}\left(C_{n, m}^{k}\right) .
$$

We may sum both sides over the cubes $C_{n, m}^{k} \in \mathscr{C}_{n, m}^{\text {int }}$ to obtain

$$
\sum_{C_{n, m}^{k} \in \mathscr{C}_{n, m}^{\text {int }}} \sum_{j=1}^{N} \int_{\phi^{k, j}}\left|\left[u_{m}^{(n)}\right](x) \cdot v(x)\right| d \mathscr{H}^{N-1}(x)=|\operatorname{tr} M| \mathscr{L}^{N}\left(\bigcup_{C_{n, m}^{k} \in \mathscr{C}_{n, m}^{\text {int }}} C_{n, m}^{k}\right) .
$$

The faces represented on the left-hand side need not include all of $J\left(u_{n, m}\right) \subset$ $\bigcup_{k=1}^{K_{n, m}} \partial C_{n, m}^{k}$, because some faces of cubes $C_{n, m}^{k} \in \mathscr{C}_{n, m}^{\text {int }}$ that are also faces of cubes $C_{n, m}^{k^{\prime}} \in \mathscr{C}_{n, m} \backslash \mathscr{C}_{n, m}^{\text {int }}$ are left out, while proper subsets $\phi_{n, m}^{k, i \pm} \cap\left(1-\frac{2}{n+2}\right) Q$ of faces $\phi_{n, m}^{k, i \pm}$ also are left out. However, for those parts of $J\left(u_{n, m}\right)$, we may use (4-1) and

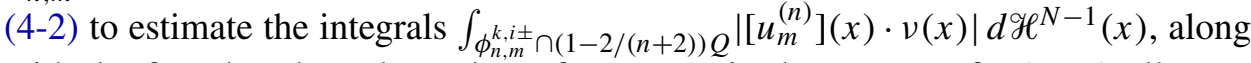
with the fact that the cubes whose faces contain these parts of $J\left(u_{n, m}\right)$ all must contain points of $\partial\left(1-\frac{2}{n+2}\right) Q$ and must together cover $\partial\left(1-\frac{2}{n+2}\right) Q$. Combining all of these contributions to $\int_{J\left(u_{n, m}\right)}\left|\left[u_{m}^{(n)}\right](x) \cdot v(x)\right| d \mathscr{H}^{N-1}(x)$, we obtain

$$
\begin{aligned}
0 & \leq \int_{J\left(u_{n, m}\right)}\left|\left[u_{m}^{(n)}\right](x) \cdot v(x)\right| d \mathscr{H}^{N-1}(x)-|\operatorname{tr} M| \mathscr{L}^{N}\left(\bigcup_{C_{n, m}^{k} \in \mathscr{C}_{n, m}^{\mathrm{int}}} C_{n, m}^{k}\right) \\
& \leq 2|\operatorname{tr} M| \mathscr{L}^{N}\left(\bigcup_{C_{n, m}^{k} \in\left(\mathscr{C}_{n, m} \backslash \mathscr{G}_{n, m}^{\mathrm{int}}\right)} C_{n, m}^{k}\right) .
\end{aligned}
$$

The factor of $2=2 N / N$ in the last expression reflects the fact that the $\mathscr{L}^{N}$-measure of some of the cubes in the collection $\mathscr{C}_{n, m} \backslash \mathscr{C}_{n, m}^{\text {int }}$ has been counted more than once but no more than $2 N$ times through the use of the bound (4-2). The relations (4-3), 
(2-2), (2-3), and (2-4) now yield the relation

$$
\begin{array}{r}
0 \leq \int_{J\left(u_{m}^{(n)}\right)}\left|\left[u_{m}^{(n)}\right](x) \cdot v(x)\right| d \mathscr{H}^{N-1}(x)-|\operatorname{tr} M| \mathscr{L}^{N}\left(\bigcup_{C_{n, m}^{k} \in \mathscr{C}_{n, m}^{\text {int }}} C_{n, m}^{k}\right) \\
\leq 2|\operatorname{tr} M| \mathscr{L}^{N}\left(\bigcup_{C_{n, m}^{k} \in\left(\mathscr{C}_{n, m} \backslash \mathscr{C}_{n, m}^{\text {int }}\right)} C_{n, m}^{k}\right)+\frac{\sqrt{N}}{m}\|M\| 2 N\left(1-\frac{2}{n+2}\right)^{N-1} \\
+C(N)\|M\|\left(1-\left(1-\frac{2}{n+2}\right)^{N}\right) .
\end{array}
$$

We in turn use (4-4) to obtain an upper bound for

$$
\int_{J\left(u_{m}^{(n)}\right)}\left|\left[u_{m}^{(n)}\right](x) \cdot v(x)\right| d \mathscr{H}^{N-1}(x) .
$$

Let $\varepsilon>0$ be given, choose $n$ so large that $C(N)\|M\|\left(1-\left(1-\frac{2}{n+2}\right)^{N}\right)<\varepsilon$, and for such an $n$, choose $m$ so large that $(\sqrt{N} / m)\|M\| 2 N\left(1-\frac{2}{n+2}\right)^{N-1}<\varepsilon$. Because $\left(1-\frac{2}{n+2}\right) Q$ has finite $\mathscr{L}^{N}$-measure, we may choose $m$ larger if necessary so that the cover $\mathscr{C}_{n, m}$ of $\left(1-\frac{2}{n+2}\right) Q$ satisfies $\mathscr{L}^{N}\left(\bigcup_{C_{n, m}^{k} \in \mathscr{C}_{n, m}} C_{n, m}^{k}\right)<$ $\mathscr{L}^{N}\left(\left(1-\frac{2}{n+2}\right) Q\right)+\varepsilon<1+\varepsilon$. Finally, because $\partial\left(1-\frac{2}{n+2}\right) Q$ has zero $\mathscr{L}^{N}$-measure and is covered by $\mathscr{C}_{n, m} \backslash \mathscr{C}_{n, m}^{\text {int }}$, we may again choose $m$ larger, if necessary, so that $2|\operatorname{tr} M| \mathscr{L}^{N}\left(\bigcup_{C_{n, m}^{k} \in\left(\mathscr{C}_{n, m} \backslash \mathscr{G}_{n, m}^{\text {int }}\right)} C_{n, m}^{k}\right)<\varepsilon$. We conclude that for $n$ and $m$ so chosen

$$
\int_{J\left(u_{m}^{(n)}\right)}\left|\left[u_{m}^{(n)}\right](x) \cdot v(x)\right| d \mathscr{H}^{N-1}(x)<|\operatorname{tr} M|(1+\varepsilon)+3 \varepsilon=|\operatorname{tr} M|+(|\operatorname{tr} M|+3) \varepsilon
$$

and, since $\varepsilon>0$ was arbitrary, that (1-27) holds.

\section{Additional explicit formulas for disarrangement densities}

Our discussion above shows that the particular choice of interfacial measure of disarrangements

$$
\int_{J(u) \cap \Omega}|[u] \cdot v| d \mathscr{H}^{N-1}
$$

for deformations $u$ of a region $\Omega \subset \mathbb{R}^{N}$ leads in both the Choksi-Fonseca relaxation scheme [1997] and in the Baía-Matias-Santos relaxation scheme [Baía et al. 2012] to the same bulk density of disarrangements

$$
\int_{\Omega}|\operatorname{tr}(\nabla g-G)| d \mathscr{L}^{N}
$$

for structured deformations $(g, G)$ of that region. Moreover, our analysis here provides an alternative to the proof of this result given in [Owen and Paroni 2015]. In that article, it was observed that replacement of $|[u] \cdot v|$ by its positive part $([u] \cdot v)^{+}=\frac{1}{2}(|[u] \cdot v|+[u] \cdot v)$ results in the replacement of $|\operatorname{tr}(\nabla g-G)|$ by its positive part $(\operatorname{tr}(\nabla g-G))^{+}=\frac{1}{2}(|\operatorname{tr}(\nabla g-G)|+\operatorname{tr}(\nabla g-G))$ in the relaxed 
bulk disarrangement density. (An analogous result holds for the negative parts, obtained by replacing "+" by "-" in the definition of the positive parts.) As pointed out in [Owen and Paroni 2015], $(\operatorname{tr}(\nabla g-G)(x))^{+}$may now be interpreted as the minimum volume fraction at a point $x \in \Omega$ that can be swept out by submacroscopic separations associated with deformations $u_{n}$ approximating the structured deformation $(g, G)$. Moreover, $(\operatorname{tr}(\nabla g-G)(x))^{-}$is the minimum volume fraction at $x$ swept out by submacroscopic switches and interpenetrations so that $|\operatorname{tr}(\nabla g-G)(x)|=(\operatorname{tr}(\nabla g-G)(x))^{+}+(\operatorname{tr}(\nabla g-G)(x))^{-}$is the minimum volume fraction swept out by submacroscopic separations, switches, and interpenetrations.

The presence of the inner product $[u] \cdot v$ in the initial interfacial density (5-1) tells us that only normal components of jumps will contribute and that alternative initial interfacial densities are required in order to capture contributions of tangential components of jumps. In the remainder of this section, we shall provide alternative initial interfacial densities that not only capture contributions of tangential components of jumps but also lead to specific formulas for the relaxed bulk disarrangement density via the "tilted cube" construction provided in Sections 2 and 4 above.

Let $a \in \mathbb{R}^{N}$ be given, and consider the replacement for (5-1)

$$
\int_{J(u) \cap \Omega}|[u] \cdot a| d \mathscr{H}^{N-1}
$$

in which the normal component $[u] \cdot v$ of the jump in $u$ is replaced by the component $[u] \cdot a$ in the direction of $a$. To again follow the relaxation scheme in [Baía et al. 2012], we let $A, B \in \mathbb{R}^{N \times N}$ be given and require not only $u \in \operatorname{SBV}\left(Q, \mathbb{R}^{N}\right)$ but also

$$
\left.u\right|_{\partial Q}=0, \quad \nabla u=B-A, \quad \mathscr{L}^{N} \text {-a.e. in } Q .
$$

We now may use the Gauss-Green formula and (5-3) to write

$$
\begin{aligned}
\int_{J(u) \cap Q}|[u] \cdot a| d \mathscr{H}^{N-1} & =\int_{J(u) \cap Q}|([u \cdot a]) v| d \mathscr{H}^{N-1} \\
& \geq\left|\int_{J(u) \cap Q}([u \cdot a]) v d \mathscr{H}^{N-1}\right| \\
& =\left|-\int_{Q} \nabla(u \cdot a) d \mathscr{L}^{N}+\int_{\partial Q}(u \cdot a) v d \mathscr{H}^{N-1}\right| \\
& =\left|-\int_{Q}(\nabla u)^{T} a d \mathscr{L}^{N}+\int_{\partial Q}(0 \cdot a) v d \mathscr{H}^{N-1}\right| \\
& =\left|(B-A)^{T} a\right| .
\end{aligned}
$$


For the "tilted-cube" construction provided in Sections 2 and 4, we replace the matrix $M$ by $B-A$, and the relation (2-6) here has the counterpart

$$
\begin{aligned}
\int_{\phi_{n, m}^{k, i+}}\left|\left[u_{m}^{(n)}\right](x) \cdot a\right| d \mathscr{H}^{N-1}(x) & =\int_{\phi_{n, m}^{k, i+}}\left|\left(\left[u_{m}^{(n)}\right](x) \cdot a\right) v(x)\right| d \mathscr{H}^{N-1}(x) \\
& =\int_{\phi_{n, m}^{k, i+}} \frac{1}{m}\left|\left((B-A) R e_{i} \cdot a\right) R e_{i}\right| d \mathcal{H}^{N-1}(x) \\
& =\frac{1}{m^{N}}\left|\left(R e_{i} \cdot(B-A)^{T} a\right) R e_{i}\right|,
\end{aligned}
$$

and this formula leads to the counterpart of (2-8)

$$
\begin{aligned}
\sum_{i=1}^{N} \int_{\phi_{n, m}^{k, i+}}\left|\left[u_{m}^{(n)}\right](x) \cdot a\right| d \mathscr{H}^{N-1}(x) & =\frac{1}{m^{N}} \sum_{i=1}^{N}\left|\left(R e_{i} \cdot(B-A)^{T} a\right) R e_{i}\right| \\
& \geq \frac{1}{m^{N}}\left|\sum_{i=1}^{N}\left(R e_{i} \cdot(B-A)^{T} a\right) R e_{i}\right| \\
& =\frac{1}{m^{N}}\left|(B-A)^{T} a\right| .
\end{aligned}
$$

The method employed in Sections 2 and 4 (where the symbol $M$ was used in place of $B-A$ ) then requires the choice of a rotation $R$ for which equality holds in the second line of (5-4). If $(B-A)^{T} a \neq 0$, we may choose $R$ to be any rotation satisfying $R e_{1}=(B-A)^{T} a /\left|(B-A)^{T} a\right|$, and this requirement is then met because $\left(R e_{i} \cdot(B-A)^{T} a\right) R e_{i}=0$ for $i=2, \ldots, N$. If $(B-A)^{T} a=0$, then $R$ can be chosen arbitrarily; for example, $R=I$ suffices.

These observations show that the analysis in Section 4 for (5-1) may be carried out step by step for the alternative initial density (5-2), provided that everywhere in Section 4 we replace $|\operatorname{tr} M|=|\operatorname{tr}(B-A)|$ by $\left|(B-A)^{T} a\right|$, the Euclidean norm of the vector $(B-A)^{T} a$. If we now define

$$
\begin{aligned}
H(A, B, a):=\inf \left\{\int_{J(u)}|[u](x) \cdot a| d \mathcal{H}^{N-1}(x): u\right. & \in S B V\left(Q ; \mathbb{R}^{N}\right), \\
& \left.\left.u\right|_{\partial Q}=0, \nabla u=B-A \text { a.e. }\right\},
\end{aligned}
$$

then our observations amount to the formula

$$
H(A, B, a)=\left|(B-A)^{T} a\right|
$$

for the relaxed bulk energy density corresponding to the initial interfacial energy (5-2) and arising from the scheme in [Baía et al. 2012]. Moreover, an argument similar to that used in establishing (1-26) shows that the formula (5-5) also holds for the relaxed bulk disarrangement density according to [Choksi and Fonseca 1997]. In 
the context of a given structured deformation $(g, G)$ on a region $\Omega$, (5-5) implies that the particular choice of initial interfacial disarrangement

$$
\int_{J(u) \cap \Omega}|[u] \cdot a| d \mathscr{H}^{N-1}
$$

for deformations $u$ of a region $\Omega \subset \mathbb{R}^{N}$ leads in both the Choksi-Fonseca relaxation scheme [1997] and in the Baía-Matias-Santos relaxation scheme [Baía et al. 2012] to the same relaxed bulk disarrangement density

$$
\int_{\Omega}\left|(\nabla g-G)^{T} a\right| d \mathscr{L}^{N}
$$

for structured deformations $(g, G)$ of that region. The integral in (5-6) represents the most economical way of introducing jumps in the direction of $a$ while approaching in the limit the given structured deformation $(g, G)$, including both jumps normal and tangential to the discontinuity surfaces of approximating deformations $u$.

We also note the formula

$$
\max _{i=1, \ldots, N} H\left(A, B, \delta_{i}\right)=\|B-A\|_{\text {row max }}
$$

where on the left $\delta_{1}, \ldots, \delta_{N}$ denote the standard basis of $\mathbb{R}^{N}$ and on the right $\|B-A\|_{\text {row max }}$ denotes the maximum of the Euclidean norms of the rows of $B-A$. The mapping $\|\cdot\|_{\text {row max }}: \mathbb{R}^{N \times N} \rightarrow \mathbb{R}$ turns out to be a norm on $\mathbb{R}^{N \times N}$, and our interpretation of the integral in (5-6) leads us to interpret the integral

$$
\int_{\Omega}\|(\nabla g-G)(x)\|_{\text {row max }} d \mathscr{L}^{N}(x)
$$

as a bulk measure of disarrangements that takes into account at each $x \in \Omega$ the direction $\delta_{i(x)}$ that maximizes the relaxed bulk energy densities $H\left(\nabla g(x), G(x), \delta_{i}\right)$ for $i=1, \ldots, N$. The bulk disarrangement density $\max _{i=1, \ldots, N} H\left(A, B, \delta_{i}\right)=$ $\|B-A\|_{\text {row max }}$ satisfies

$$
\begin{array}{r}
\max _{i=1, \ldots, N} H\left(A, B, \delta_{i}\right) \leq \inf \left\{\max _{i=1, \ldots, N} \int_{J(u)}\left|[u](x) \cdot \delta_{i}\right| d \mathscr{H}^{N-1}(x): u \in S B V\left(Q ; \mathbb{R}^{N}\right),\right. \\
\left.\left.u\right|_{\partial Q}=0, \nabla u=B-A \text { a.e. }\right\}
\end{array}
$$

and need not be the relaxed bulk energy density corresponding to the initial interfacial energy $\max _{i=1, \ldots, N} \int_{J(u)}\left|[u](x) \cdot \delta_{i}\right| d \mathscr{H}^{N-1}(x)$.

\section{Acknowledgments}

The authors warmly thank the Center for Nonlinear Analysis (NSF grants numbers DMS-0405343 and DMS-0635983) at Carnegie Mellon University and the 
Centre for Mathematical Analysis, Geometry, and Dynamical Systems (FCT grant UID/MAT/04459/2013) at Instituto Superior Técnico, where this research was carried out. The research of Barroso, Matias, and Morandotti was partially supported by the Fundação para a Ciência e a Tecnologia (Portuguese Foundation for Science and Technology) through the CMU-Portugal Program under grant FCTUTA_CMU/MAT/0005/2009 “Thin Structures, Homogenization, and Multiphase Problems". The research of Barroso was partially supported by the Fundação para a Ciência e a Tecnologia through grant PEst_OE/MAT/UI0209/2013. The research of Morandotti was partially supported by the European Research Council through the ERC Advanced Grant "QuaDynEvoPro", grant agreement 290888. Morandotti is a member of the Progetto di Ricerca GNAMPA-INdAM 2015 "Fenomeni critici nella meccanica dei materiali: un approccio variazionale" (INdAM-GNAMPA Project 2015 "Critical phenomena in the mechanics of materials: a variational approach").

\section{References}

[Baía et al. 2011] M. Baía, J. Matias, and P. M. Santos, "A survey on structured deformations", São Paulo J. Math. Sci. 5:2 (2011), 185-201.

[Baía et al. 2012] M. Baía, J. Matias, and P. M. Santos, "A relaxation result in the framework of structured deformations in a bounded variation setting", Proc. Roy. Soc. Edinburgh Sect. A 142:2 (2012), 239-271.

[Choksi and Fonseca 1997] R. Choksi and I. Fonseca, "Bulk and interfacial energy densities for structured deformations of continua", Arch. Rational Mech. Anal. 138:1 (1997), 37-103.

[Choksi et al. 1999] R. Choksi, G. Del Piero, I. Fonseca, and D. Owen, "Structured deformations as energy minimizers in models of fracture and hysteresis", Math. Mech. Solids 4:3 (1999), 321-356.

[Ciblak and Lipkin 1998] N. Ciblak and H. Lipkin, "Orthonormal isotropic vector bases", in Proceedings of DETC'98: 1998 ASME Design Engineering Technical Conferences (Atlanta, 1998), ASME International, New York, 1998.

[Del Piero and Owen 1993] G. Del Piero and D. R. Owen, "Structured deformations of continua", Arch. Rational Mech. Anal. 124:2 (1993), 99-155.

[Del Piero and Owen 1995] G. Del Piero and D. R. Owen, "Integral-gradient formulae for structured deformations", Arch. Rational Mech. Anal. 131:2 (1995), 121-138.

[Deseri and Owen 2003] L. Deseri and D. R. Owen, "Toward a field theory for elastic bodies undergoing disarrangements", J. Elasticity 70:1-3 (2003), 197-236.

[Kröner 1958] E. Kröner, Kontinuumstheorie der Versetzungen und Eigenspannungen, Ergebnisse angewandten Math. 5, Springer, Berlin, 1958.

[Matias 2007] J. Matias, "Differential inclusions in $\operatorname{SBV}_{0}(\Omega)$ and applications to the calculus of variations", J. Convex Anal. 14:3 (2007), 465-477.

[Matias and Santos 2014] J. Matias and P. M. Santos, "A dimension reduction result in the framework of structured deformations", Appl. Math. Optim. 69:3 (2014), 459-485.

[Nye 1953] J. F. Nye, "Some geometrical relations in dislocated crystals", Acta Metall. 1:2 (1953), $153-162$. 
[Owen and Paroni 2000] D. R. Owen and R. Paroni, "Second-order structured deformations", Arch. Ration. Mech. Anal. 155:3 (2000), 215-235.

[Owen and Paroni 2015] D. R. Owen and R. Paroni, "Optimal flux densities for linear mappings and the multiscale geometry of structured deformations", Arch. Ration. Mech. Anal. 218:3 (2015), $1633-1652$.

[Šilhavý 2015] M. Šilhavý, "On the approximation theorem for structured deformations from $B V(\Omega)$ ", Math. Mech. Complex Syst. 3:1 (2015), 83-100.

[Šilhavý 2016] M. Šilhavý, "Relaxed disarrangements densities for structured deformations”, preprint IM-2016-53, Institute of Mathematics, CAS, 2016, Available at http://tinyurl.com/silhavy.

Received 8 Jul 2016. Accepted 31 Oct 2016.

ANA CRISTINA BARROSO: acbarroso@ciencias.ulisboa.pt

Departamento de Matemática and Centro de Matemática, Aplicações Fundamentais e Investigação Operacional, Faculdade de Ciências da Universidade de Lisboa, Campo Grande, Edifício C6, Piso 1, 1749-016 Lisboa, Portugal

José Matias: jose.c.matias@tecnico.ulisboa.pt

Departamento de Matemática, Instituto Superior Técnico, Avenida Rovisco Pais 1, 1049-001 Lisboa, Portugal

MARCO MORANDOTTI: marco.morandotti@ma.tum.de

Fakultät für Mathematik, Technische Universität München, Boltzmannstraße 3, 85748 Garching bei München, Germany

DAVID R. OWEN: do04@andrew. cmu .edu

Department of Mathematical Sciences, Carnegie Mellon University, 5000 Forbes Avenue, Pittsburgh, PA 15213, United States 